\title{
Color Sorting of Coated Brassica Seeds by Fluorescent Sinapine Leakage to Improve Germination
}

\author{
A.G. Taylor ${ }^{1}$, D.B. Churchill' ${ }^{2}$ S.S. Lee ${ }^{3}$, D.M. Bilsland ${ }^{4}$, and T.M. Cooper ${ }^{5}$ \\ Department of Horticultural Sciences, New York State Agricultural Experiment Station, Cornell University, \\ Geneva, NY 14456

\section{U.S. Department of Agriculture-Agricultural Research Service, National Forage Seed Production Research Center, Corvallis, OR 97331}

Additional index words. seed conditioning, sinapine, cabbage, broccoli, cauliflower

\begin{abstract}
Color sorting was performed to upgrade seed quality by removal of fluorescent coated seeds. The fluorescent coating was attributed to sinapine leakage from nonviable seeds. Nine seedlots, three seedlots each of cabbage ( Brassica oleracea L. Capitata group), broccoli, and cauliflower ( B. oleracea L. Botrytis group) were custom coated. Seed samples were pretreated before coating with or without $1.0 \% \mathrm{NaOCl}$ for 10 minutes to enhance leakage. All samples revealed a percentage of seeds with fluorescence. The light emission from selected fluorescent and nonfluorescent coated seeds was quantified by fiber-optic spectrophotometry. Fluorescence was expressed from 400 to $560 \mathrm{~nm}$, with peak emission being from 430 to $450 \mathrm{~nm}$. These data confirmed our visual interpretation of blue-green fluorescence. The ratio of light emission from fluorescent compared to nonfluorescent coated seeds ranged from 4.5 to $\mathbf{7 . 0}$ for all samples and averaged 5.7. An ultraviolet (UV) color sorter was employed to separate fluorescent (reject) from nonfluorescent (accept) coated seeds. The percentage of nonfluorescent coated seeds (averaged over seedlot and $\mathrm{NaOCl}$ pretreatment) before and after sorting was $\mathbf{8 9 . 5 \%}$ and $\mathbf{9 5 . 9 \%}$, respectively. Therefore, color sorting was able to remove a high percentage of fluorescent coated seeds with an average loss (rejection of nonfluorescent coated seeds) of $6 \%$. An increase in the percent germination was recorded in eight of the nine seedlots following color sorting, and the greatest improvement was obtained with seedlots of medium quality. Germination of three medium quality lots was increased by 10 to 15 percentage points. The average increase in germination with or without $\mathrm{NaOCl}$ pretreatment was 8.2 and 5.5 percentage points, respectively. In conclusion, the germination of Brassica seedlots could be improved by separating (removing) fluorescent from nonfluorescent coated seeds. UV color sorting technology was employed to demonstrate that seed conditioning could be conducted on a commercial basis to upgrade seed quality.
\end{abstract}

Seed quality is composed of several components, including the percent germination, purity, vigor, and pathological condition of a seedlot (Copeland and McDonald, 1985). Our discussion will focus on the percent germination of seedlots and its importance on seedling establishment. The maximum potential for seedling emergence is governed by the percent germination, as a seedlot with $80 \%$ germination can not be expected to have $>80 \%$ seedling emergence. The goal is to increase the percent germination of a seedlot and, thereby, increase seedling establishment potential.

Seed conditioning has been conducted to remove contaminants from seeds including weed seeds, other crop seeds, and inert matter (Brandenburg, 1977; Brandenburg and Park, 1977). Seed conditioning has also been employed to grade seeds by physical traits such as length, width, or thickness (Harmond et al., 1968). The ability to upgrade the germination of seedlots based on physical characteristics alone appears to be limited. The concept of combin-

Received for publication 2 Apr. 1992. Accepted for publication 3 Nov. 1993. This research was partially supported by a grant from Seed Systems, Gilroy, Calif. We acknowledge statistical advice from K.S. Yourstone. The cost of publishing this paper was defrayed in part by the payment of page charges. Under postal regulations, this paper therefore must be hereby marked advertisement solely to indicate this fact.

Associate Professor of Seed Science and Technology.

${ }^{2}$ Agricultural Engineer, U.S. Dept. of Agriculture, Agricultural Research Service, National Forge Seed Production Research Center, Corvallis, OR 97331.

${ }^{3}$ Visiting Scientist.

${ }^{4}$ Senior Faculty Research Assistant, Bioresource Engineering Dept., Oregon State Univ., Corvallis.

${ }^{5}$ Former Senior Research Assistant, Bioresource Engineering Dept., Oregon State Univ., Corvallis. ing seed conditioning by physical characteristics with physiological characteristics associated with seed viability could provide the opportunity to upgrade seedlot germination.

Physiological seed treatments or seed priming have been employed to enhance the rate of germination (Bradford, 1986; Taylor and Harman, 1990). Seeds were first hydrated under controlled environmental conditions followed by dehydration. Seeds treated in such a manner may be coated, if desired, stored, and eventually sown. An additional advantage of these treatments was that seeds attain a high moisture content and, therefore, become physiologically active. During this period, physiological differences between viable and nonviable seeds may be expressed.

Phenolic or aromatic choline esters have been found in seeds of the Brassicaceae, and the predominant phenolic choline ester was sinapine (3, 5-dimethoxy -4- hydroxycinnamoylcholine) (Bouchereau et al., 1991; Clausen et al., 1985). Sinapine has been shown 10 leak preferentially from nonviable Brassica seeds after imbibition (Taylor et al., 1988). This compound is fluorescent and is yellow at high $\mathrm{pH}$ (210). Sinapine leakage was found to be a better predictor of seed viability than electrolyte leakage and has been developed as a rapid seed viability test (Hill et al., 1988).

A novel coating system has been developed to exploit sinapine leakage and upgrade seedlot quality in the Brassicaceae (Taylor et al., 1990). Seeds were first hydrated, which would result in leakage from nonviable seeds, and then were coated with an adsorbent consisting of cellulose with sand. The coated seeds are then dried, examined under UV light, and manually sorted into two fractions:

Abbreviation: PMT, photomultiplier tubes. 


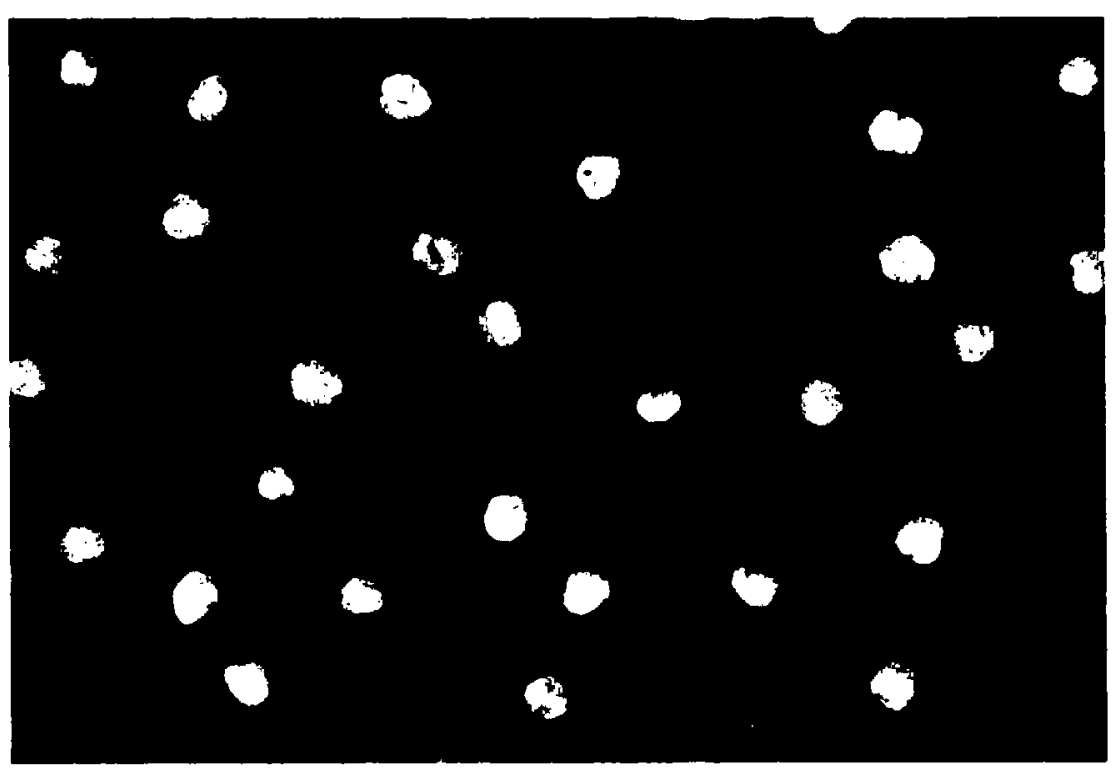

Fig. 1. A photograph of coated Brassica seeds illustrating a portion with fluorescent coatings.

fluorescent and nonfluorescent. Three horticultural Brassicas, one agronomic Brassica, and one flower species were studied with this upgrading system (Taylor et al., 1991). Sowing the nonfluorescent seeds resulted in a greater percent normal seedlings than sowing noncoated seeds in all seedlots. The fluorescent fractions contained a high percentage of dead seeds and seeds that produced abnormal seedlings.

Commercial scale application of this technology requires a rapid technique to separate the fluorescent from the nonfluorescent seeds. Color sorters have been employed to separate seeds based on differences in color or light/dark characteristics (Brandenburg and Park, 1977). Color sorters singulate seed and detect the light reflectance on a single seed basis. Seeds were rejected by an electrically operated air valve, while good (accept) seeds pass through the equipment (Cabrera and Diaz, 1991).

The objective of this project was to study color sorting of fluorescent coated Brassica seeds. Research was performed on nine seedlots; three seedlots each of cabbage, broccoli, and cauliflower. The fluorescent emission spectrum was characterized, and color sorter efficacy was determined. Upgrading of seed quality was determined by the standard germination test of nonsorted and sorted nonfluorescent fractions.

\section{Materials and Methods}

Seedlots, NaOCl pretreatment, and coating. Three crop seeds were studied: cabbage, broccoli, and cauliflower. Three seedlots of each crop were obtained from commercial vendors: cabbage 'Brunswick', 'Copenhagen Market', and 'Ruby Ball'; broccoli 'Medium Late 423', 'Premium Crop', and 'Shogun'; and cauliflower 'Blue Diamond', 'Dok Elgon', and cauliflower 'Starbrite $\mathrm{Y}^{\prime}$.

A sample from each seedlot was pretreated with $1.0 \% \mathrm{NaOCl}$ for $10 \mathrm{~min}$, and other samples were not pretreated before coating. The purpose of the $\mathrm{NaOCl}$ pretreatment was to enhance the sinapine leakage rate (Taylor et al., 1993). Nonpretreated and $\mathrm{NaOCl}$ pretreated seeds of each seedlot were coated by Seed Systems (Gilroy, Calif.), and the coating formulation was based on earlier developments at Geneva (Taylor et al., 1990). A sample of coated seeds was illuminated with UV light and photographed.
Fluorescence emission. The purpose of this study was to quantify the fluorescent emission from coated seeds using fiberoptic spectrophotometry (Berlage et al., 1989). A coated sample of each seedlot and treatment was manually separated into two fractions: fluorescent and nonfluorescent. Fifteen coated seeds were placed in a custom-designed sample holder and irradiated with UV light from a $45^{\circ}$ angle to the detector. The UV light was produced by a $50 \mathrm{~W}$ source with a $365 \mathrm{~nm}$ wavelength maximum emission (model 125120, Instrument Technology, Westfield, Mass.). The fluorescence emitted from the seed sample was detected by a UV-VIS 6 to 1 probe attached to a fiber-optic spectrophotometer (model 100, Guided Wave, El Dorado Hills, Calif.). The fiber-optic spectrophotometer had been upgraded to a model 150 and employed silicon and germanium detectors. Three data readings were taken with two dwell cycles in $4 \mathrm{~nm}$ increments. The fluorescence spectrum was initially characterized from 350 to $650 \mathrm{~nm}$. The percent transmittance was converted to emission ratio, or the ratio of light emitted from the sample compared to the blank; i.e., $100 \% \mathrm{~T}=1.0$ emission ratio and $600 \% \mathrm{~T}=6.0$ emission. Based on the spectral distribution, the area under the curve was determined from 400 to $560 \mathrm{~nm}$ for all samples, and the derived

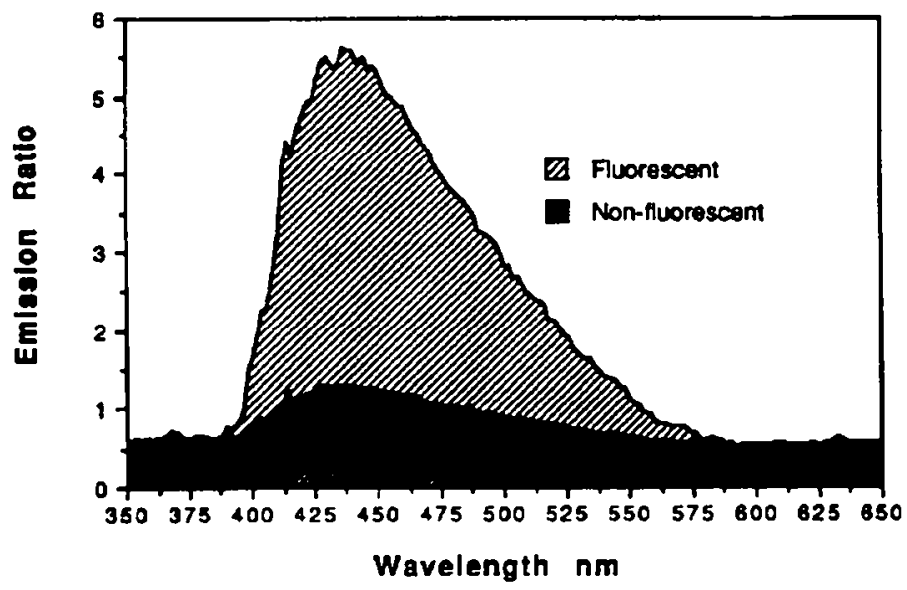

Fig. 2. The fluorescence spectrum (light emission) from fluorescent and nonfluorescent coated Brassica sceds. 
value was unitless. There were four replicate samples per treatment and standard errors were calculated.

Color sorter. An Icore model 5141 UV color sorter (ScancoreSortex, Union City, Calif.) was employed to separate fluorescent from nonfluorescent coated seeds. The feed rate was adjusted to deliver 40 to 50 seeds per second. The feed rollers were adjusted at a $45^{\circ}$ angle to singulate seeds before the detection/rejection section of the unit. Seeds were irradiated by fluorescent tubes within the detection head of the unit. Three 931B photomultiplier tubes (PMT) with a spectral range of 300 to $660 \mathrm{~nm}$ were mounted at $120^{\circ}$ angle from each other. The color sorter had "or" logic, and if any one PMT detected light, it would reject the seed. No filters were used with the PMTs, and slit size (aperture) was $2.3 \mathrm{~mm}$. Seeds were rejected with an air solenoid operated at $400 \mathrm{kPa}$ with a4 to $5 \mathrm{msec}$ pulse duration. The light sensitivity of the reject mode was determined empirically and correlated with the fiber-optic spectrophotometer values.

There were four replications of $50 \mathrm{~g}$ each per treatment used for each seedlot. A 10-g sample was removed and classified as nonsorted, and the remaining $40 \mathrm{~g}$ were sorted with the color sorter. Efficacy of the color sorting operation was verified by visual inspection under UV light. The percent nonfluorescent seeds was determined before and after sorting in the good-accept fraction. The percent nonfluorescent seeds rejected were calculated as the percent loss. Standard errors were calculated for each characteris- tic of each seedlot and treatment. A standard germination test (AOSA, 1988) was conducted on the nonsorted and sorted nonfluorescent (accept) fractions. An arcsin transformation was performed, and data were analyzed within seedlots as a $2 \times 2$ factorial in a completely randomized design.

\section{Results}

Fluorescence emission. Coated seeds were photographed under UV light and a percentage of them revealed fluorescence (Fig. 1). The fluorescence emission was quantified from selected fluorescent and nonfluorescent coated seeds with a fiber-optic spectrophotometer showing light emission (Fig. 2). The fluorescent coated seeds emission was $>1.0$ at wavelengths between 400 to 560 $\mathrm{nm}$. The maximum emission ranged from ca. 430 to $450 \mathrm{~nm}$, and the emission ratio exceeded 5.0 in this region. Emission from the nonfluorescent coated seeds was $>1.0$ between 410 to $490 \mathrm{~nm}$, but did not exceed an emission ratio of 1.4.

The fluorescence was quantified from the nine seedlots with and without the $\mathrm{NaOCl}$ pretreatment (Table 1). The data, which are unitless, provided a relative method to compare the emitted light from selected fluorescent and nonfluorescent seeds. The emission $\left(\times 10^{3}\right)$ from fluorescent and nonfluorescent coated seeds differed between treatments and ranged from 41.5 to 90.7 and 7.4 to 18.4, respectively. The average emission $\left(\times 10^{3}\right)$ from all fluorescent and

\begin{tabular}{|c|c|c|c|c|c|c|}
\hline \multirow[b]{2}{*}{ Crop } & \multirow[b]{2}{*}{ Cultivar } & \multirow[b]{2}{*}{$\mathrm{NaOCl}$} & \multicolumn{2}{|c|}{ Light emission ${ }^{2}$} & \multirow[b]{2}{*}{ Ratio } & \multirow{2}{*}{$\begin{array}{l}\text { Color sorte } \\
\text { sensitivity }\end{array}$} \\
\hline & & & Nonfluorescent & Fluorescent & & \\
\hline \multirow[t]{6}{*}{ Cabbage } & Brunswick & - & $7.4 \pm 0.2$ & $41.5 \pm 0.7$ & $5.6 \pm 0.2$ & $\overline{72}$ \\
\hline & & + & $10.3 \pm 0.2$ & $58.8 \pm 3.2$ & $5.7 \pm 0.2$ & 67 \\
\hline & Copenhagen Market & - & $9.3 \pm 0.2$ & $55.7 \pm 2.9$ & $6.0 \pm 0.4$ & 67 \\
\hline & & + & $7.6 \pm 0.1$ & $45.1 \pm 5.1$ & $5.9 \pm 0.7$ & 70 \\
\hline & Ruby Ball & - & $11.4 \pm 0.3$ & $66.9 \pm 2.2$ & $5.9 \pm 0.3$ & 62 \\
\hline & & + & $10.2 \pm 0.2$ & $60.4 \pm 2.4$ & $5.9 \pm 0.3$ & 62 \\
\hline \multirow[t]{6}{*}{ Broccoli } & Medium Late 423 & - & $13.4 \pm 1.0$ & $79.5 \pm 5.1$ & $6.0 \pm 0.6$ & 65 \\
\hline & & + & $12.2 \pm 0.1$ & $76.1 \pm 4.4$ & $6.3 \pm 0.3$ & 65 \\
\hline & Premium Crop & - & $18.4 \pm 1.0$ & $90.7 \pm 3.3$ & $5.0 \pm 0.4$ & 50 \\
\hline & & + & $15.3 \pm 0.5$ & $86.3 \pm 3.4$ & $5.6 \pm 0.2$ & 60 \\
\hline & Shogun & - & $14.6 \pm 0.7$ & $84.1 \pm 4.8$ & $5.8 \pm 0.5$ & 57 \\
\hline & & + & $11.9 \pm 0.5$ & $68.7 \pm 4.3$ & $5.7 \pm 0.1$ & 55 \\
\hline \multirow[t]{6}{*}{ Cauliflower } & Dok Elgon & - & $9.6 \pm 0.4$ & $51.5 \pm 2.6$ & $5.4 \pm 0.4$ & 65 \\
\hline & & + & $8.0 \pm 0.5$ & $45.7 \pm 3.5$ & $5.7 \pm 0.4$ & 70 \\
\hline & Starbrite & - & $9.5 \pm 1.0$ & $65.2 \pm 5.0$ & $7.0 \pm 0.6$ & 70 \\
\hline & & + & $11.5 \pm 0.3$ & $55.2 \pm 2.3$ & $4.8 \pm 0.1$ & 75 \\
\hline & Blue Diamond & - & $9.6 \pm 0.4$ & $43.7 \pm 7.3$ & $4.5 \pm 0.6$ & 70 \\
\hline & & + & $9.7 \pm 0.4$ & $41.7 \pm 1.8$ & $4.9 \pm 0.3$ & 70 \\
\hline \multirow[t]{2}{*}{ Mean } & & - & 11.5 & 64.3 & 5.7 & 64 \\
\hline & & + & 10.7 & 59.8 & 5.6 & 66 \\
\hline Grand mean & & & 11.1 & 62.0 & 5.7 & 65 \\
\hline
\end{tabular}

${ }^{2}$ The units are dimensionless; values have been multiplied by 1000 . 
Table 2. Percentage of seeds with nonfluorescent coatings before and after color sorting and percent loss.

\begin{tabular}{|c|c|c|c|c|c|}
\hline \multirow[b]{3}{*}{ Crop } & \multirow[b]{3}{*}{ Cultivar } & \multirow[b]{3}{*}{$\mathrm{NaOCl}$} & \multicolumn{3}{|c|}{ Seed $(\%)$} \\
\hline & & & \multirow{2}{*}{$\frac{\text { Before sorting }}{\text { Nonfluorescent }}$} & \multicolumn{2}{|c|}{ After sorting } \\
\hline & & & & Nonfluorescent & Loss \\
\hline \multirow[t]{6}{*}{ Cabbage } & Brunswick & - & $93.5 \pm 1.6$ & $96.0 \pm 1.0$ & $8 . \overline{4} \pm 0.3$ \\
\hline & & + & $81.3 \pm 1.5$ & $90.5 \pm 0.6$ & $6.9 \pm 0.3$ \\
\hline & Copenhagen Market & - & $90.8 \pm 1.1$ & $91.8+1.0$ & $2.9 \pm 0.3$ \\
\hline & & + & $84.8 \pm 1.9$ & $89.5 \pm 1.4$ & $7.7 \pm 0.4$ \\
\hline & Ruby Ball & - & $87.3 \pm 1.1$ & $97.5 \pm 0.3$ & $5.6 \pm 0.5$ \\
\hline & & + & $85.3 \pm 1.1$ & $96.3 \pm 1.5$ & $6.3 \pm 0.2$ \\
\hline \multirow[t]{6}{*}{ Broccoli } & Medium Late 423 & - & $96.0 \pm 0.7$ & $99.8 \pm 0.3$ & $2.3 \pm 0.2$ \\
\hline & & + & $96.3 \pm 0.5$ & $98.5 \pm 0.5$ & $2.9 \pm 0.3$ \\
\hline & Premium Crop & - & $86.3 \pm 2.9$ & $97.8 \pm 0.6$ & $3.2 \pm 0.6$ \\
\hline & & + & $88.0 \pm 2.0$ & $97.8 \pm 0.6$ & $8.7 \pm 0.7$ \\
\hline & Shogun & - & $77.5 \pm 2.8$ & $95.8 \pm 1.1$ & $4.7 \pm 0.6$ \\
\hline & & + & $77.5 \pm 1.2$ & $92.0 \pm 1.8$ & $5.3 \pm 0.7$ \\
\hline \multirow[t]{6}{*}{ Cauliflower } & Dok Elgon & - & $90.3 \pm 1.8$ & $96.8 \pm 1.3$ & $13.0 \pm 0.6$ \\
\hline & & + & $90.3 \pm 1.1$ & $92.3 \pm 1.7$ & $10.2 \pm 0.9$ \\
\hline & Starbrite Y & - & $96.5 \pm 1.0$ & $98.5 \pm 0.9$ & $3.1 \pm 0.2$ \\
\hline & & + & $95.0 \pm 1.4$ & $98.5 \pm 0.6$ & $7.9 \pm 0.4$ \\
\hline & Blue Diamond & - & $97.8 \pm 0.6$ & $98.8 \pm 0.3$ & $3.2 \pm 0.1$ \\
\hline & & + & $96.8 \pm 0.5$ & $97.5 \pm 1.2$ & $4.4 \pm 0.2$ \\
\hline \multirow[t]{2}{*}{ Mcan } & & - & 90.7 & 97.0 & 5.2 \\
\hline & & + & 88.4 & 94.8 & 6.7 \\
\hline Grand mean & & & 89.5 & 95.9 & 5.9 \\
\hline
\end{tabular}

nonfluorescent coated seeds was 62.0 and 11.1, respectively. The $\mathrm{NaOCl}$ pretreatment affected emission for certain seedlots, but there was no consistent trend among seedlots. Overall, $\mathrm{NaOCl}$ slightly increased light emission from fluorescent and nonfluorescent coated seeds compared to nonpretreated seeds. The ratio of emitted light from fluorescent to nonfluorescent coated seeds ranged from 4.5 to 7.0 and averaged 5.7.

Color sorter. The UV color sorter sensitivity was adjusted on an empirical basis for each treatment. This was accomplished by selecting known fluorescent or nonfluorescent coated seeds, passing them through the sorter singly and adjusting the sensitivity for optimum separation. Therefore, the sensitivity setting may differ among and within seedlots (Table 1). The UV color sorter sensitivity level was negatively correlated with both fluorescent and nonfluorescent values obtained from the fiber-optic spectrophotometer, $r=-0.78^{* *}$.

The efficacy of the UV color sorter was verified by visual inspection. All seedlots studied contained a percentage of fluorescent coated seeds that was attributed to sinapine leakage into the coated seeds from poor quality seeds (Taylor et al., 1991). The percent nonfluorescent coated seeds differed among seedlots before sorting and ranged from 77.5 to 97.8 with an overall average of 90 (Table 2). The $\mathrm{NaOCl}$ pretreatment decreased the percent nonfluorescent coated seeds only in cabbage 'Brunswick' and 'Copenhagen Market' before sorting. After sorting, the percent nonfluorescent coated seeds ranged from 89.5 to 99.8 , and the $\mathrm{NaOCl}$ decreased the percent nonfluorescent coated seeds in three of the nine seedlots (cabbage 'Brunswick', broccoli 'Shogun', and cauliflower 'Dok Elgon'). There was an average increase of six percentage points in nonfluorescent coated seeds due to sorting (89.5 vs. 95.9), that was attributed to removal of fluorescent seeds by the color sorter. Loss was $<13 \%$ and averaged $6 \%$.

A standard germination test was performed to study the interaction of sorting and $\mathrm{NaOCl}$. The analysis was performed on normal seedlings for each seedlot separately. Sorting the coated seed resulted in a significant improvement in the percent germination in eight of the nine seedlots (Table 3). Only cauliflower 'Dok Elgon' did not show a significant difference due to sorting. The average percent germination of the nine seedlots before sorting was 82. After sorting, the germination with or without $\mathrm{NaOCl}$ pretreatment was $90.6 \%$ and $87.8 \%$. respectively. A significant improvement in germination was measured in medium quality seedlots. The initial germination was $67.1 \%$ for cabbage 'Ruby Ball', 70.3\% for broccoli 'Premium Crop', and 74.9\% for broccoli 'Shogun' (averaged over $\mathrm{NaOCl}$ treatments), compared to $81.8 \%$. $84.4 \%$. and $85.3 \%$, respectively, after sorting. $\mathrm{NaOCl}$ resulted in a slight decrease in germination in broccoli 'Medium Late 423', while cabbage 'Ruby Ball' exhibited an improvement of 14 percentage points (averaged over sorting). Overall, $\mathrm{NaOCl}$ pretreatment resulted in a three percentage point increase after sorting 


\begin{tabular}{|c|c|c|c|c|c|c|c|c|}
\hline \multirow[b]{2}{*}{ Crop } & \multirow[b]{2}{*}{ Cultivar } & \multirow[b]{2}{*}{$\mathrm{NaOCl}$} & \multicolumn{2}{|c|}{ Germination (\%) } & \multicolumn{4}{|c|}{ Mean squares and level of significance } \\
\hline & & & Nonsorted & Sorted & Sorted & $\mathrm{NaOCl}$ & $\begin{array}{c}\text { Sorted } \\
\times \mathrm{NaOCl}\end{array}$ & Residual \\
\hline \multirow[t]{6}{*}{$\overline{\text { Cabbage }}$} & Brunswick & - & 78.3 & 79.3 & $0.0122 *$ & $<0.0001^{\mathrm{SS}}$ & $0.0075^{\circ}$ & 0.0006 \\
\hline & & + & 74.8 & 82.8 & & & & \\
\hline & Copenhagen Market & - & 91.3 & 95.0 & $0.0409^{* *}$ & $<0.0001^{\mathrm{Ns}}$ & $0.0023^{\mathrm{Ns}}$ & 0.0015 \\
\hline & & + & 89.8 & 96.0 & & & & \\
\hline & Ruby Ball & - & 59.3 & 75.8 & $0.1186^{\circ *}$ & $0.1066^{\circ *}$ & $0.0001^{\mathrm{NS}}$ & 0.0007 \\
\hline & & + & 75.0 & 87.8 & & & & \\
\hline \multirow[t]{6}{*}{ Broccoli } & Medium Late 423 & - & 88.8 & 93.0 & $0.0074^{* *}$ & $0.0064^{*}$ & $0.0040^{\mathrm{*s}}$ & 0.0011 \\
\hline & & + & 88.3 & 89.0 & & & & \\
\hline & Premium Crop & - & 73.0 & 82.0 & $0.1174^{\circ *}$ & $<0.0001^{\mathrm{vs}}$ & $0.0158^{\circ *}$ & 0.0006 \\
\hline & & + & 67.5 & 86.8 & & & & \\
\hline & Shogun & - & 74.5 & 84.0 & $0.0697^{* *}$ & $0.0019^{\mathrm{ns}}$ & $0.0007^{\mathrm{Ns}}$ & 0.0011 \\
\hline & & + & 75.3 & 86.5 & & & & \\
\hline \multirow[t]{6}{*}{ Cauliflower } & Dok Elgon & - & 89.3 & 91.5 & $0.0022^{\mathrm{NS}}$ & $0.140^{\mathrm{ws}}$ & $0.0007^{\mathrm{Ns}}$ & 0.0034 \\
\hline & & + & 93.0 & 93.8 & & & & \\
\hline & Starbrite Y & - & 94.3 & 94.8 & $0.0264^{\circ}$ & $0.0009^{\mathrm{Ns}}$ & $0.0200^{\circ}$ & 0.0022 \\
\hline & & + & 89.8 & 96.8 & & & & \\
\hline & Blue Diamond & - & 92.3 & 94.5 & $0.381^{*}$ & $0.0028^{\mathrm{Ns}}$ & $0.0095^{\circ}$ & 0.0016 \\
\hline & & + & 87.8 & 9.5 .5 & & & & \\
\hline \multirow[t]{2}{*}{ Mean } & & - & 82.3 & 87.8 & & & & \\
\hline & & + & 82.4 & 90.6 & & & & \\
\hline Grand mean & & & 82.4 & 89.2 & & & & \\
\hline
\end{tabular}

$\overline{N S,{ }^{*},{ }^{* *}}$ Nonsignificant or significant at $P=0.05$ or 0.01 , respectively.

than did nonpretreated seeds. Four seedlots, cabbage 'Brunswick', broccoli 'Premium Crop', and cauliflower 'Starbrite Y' and 'Blue Diamond' revealed a significant interaction with sorting and $\mathrm{NaOCl}$, and the same seedlots also exhibited a highly significant difference due to sorting. The percent of the treatment mean square attributed to the interaction was $38 \%$ for cabbage 'Brunswick', $12 \%$ for broccoli 'Premium Crop', $42 \%$ for cauliflower 'Starbrite Y', and $19 \%$ for cauliflower 'Blue Diamond', compared to $62 \%$. $88 \%, 56 \%$, and $76 \%$ for the sorting effect, respectively.

\section{Discussion}

The concept of this project was to combine physiological presowing treatments with seed conditioning. Seed coating technology was necessary to bridge these two components into a complete system. Physiological presowing treatments are treatments that involve hydration and dehydration of seeds under controlled environmental conditions for a specified period of time (Taylor and Harman, 1990). One consequence of hydrating seeds is that the nonviable Brassica seeds would preferentially leak sinapine into the imbibing medium (Taylor et al., 1988). Sinapine would normally be discarded at the completion of the hydration phase in a conventional physiological seed treatment. However, it could be exploited as a biochemical marker to detect seed viability.

Seed coating or pelleting technologies consist of coating indi- vidual particles (seeds) in a batch process (Scott, 1989; Taylor and Ham-tan, 1990). Seeds may be coated immediately after hydration without dehydrating them (Kaufman, 1991). An adsorbent material could be used as the filler in the coating to trap sinapine (Taylor et al., 1991). After coating, the coated seeds could be dried to decrease moisture content to facilitate seed handling and prolong storage.

Finally, the compound that leaks must be detected physically. In our system, the sinapine was fluorescent, thereby providing a natural indicator for its presence. Seed conditioning equipment, such as the UV color sorter, could be employed to separate seeds with fluorescent coated seeds from nonfluorescent coated seeds. The UV color sorter would upgrade seed lot quality and demonstrate the commercial feasibility of this system.

The fluorescence expressed on a single seed basis was readily observed with UV illumination (Fig. 1). The fluorescence spectrum revealed light emission from 400 to $560 \mathrm{~nm}$ (Fig. 2). This physical description verifies our visual interpretation that the fluorescence was blue-green (Taylor et al., 1988). The fluorescence emission varied with seedlot (Table 1) and may be attributed to differences in leakage rate, sinapine content, and coating characteristics. The ratio of light emitted from selected fluorescent and nonfluorescent coated seeds ranged from 7.0 to 4.5 with an average of 5.7. Therefore, large differences in leakage as determined by light emission was consistently observed among seedlots. 
The $\mathrm{NaOCl}$ pretreatment was intended to accelerate leakage and, therefore, enhance fluorescence (Taylor et al., 1993). In preliminary studies, the $\mathrm{NaOCl}$ pretreatment was shown to accelerate leakage from heat-killed and aged seeds. Therefore, greater fluorescence emission was expected from pretreated seeds. Coated seeds with the $\mathrm{NaOCl}$ pretreatment had, on average, less fluorescence emission than nonpretreated seeds (Table 1). This reduction may have been attributed to oxidation of sinapine.

Color sorter efficacy was verified by visual inspection under UV light. It was difficult to classify coated seeds as either fluorescent or nonfluorescent due to several reasons. There was a percentage of coated seeds that exhibited partial or incomplete fluorescence. The light emission from these coated seeds would be less than those selected with uniform fluorescence. The degree of observed fluorescence was a subjective evaluation, and the intensity of fluorescence was determined by the UV light source and proximity of the source to the sample.

An increase in percent nonfluorescent coated seeds was generally obtained after color sorting (Table 2). The color sorter was thus able to remove a significant portion of fluorescent coated seeds. A relatively small percentage loss was encountered with the color sorting operation. The loss averaged 6\%, and some loss is customary for any seed conditioning operation. The percent improvement (i.e., increase in nonfluorescent coated seeds) and loss are directly controlled by the sensitivity setting on the color sorter. A higher percentage improvement would be accomplished with a concomitant increase in loss and vice versa. A sample could be passed through the color sorter twice to further decrease the percent fluorescent coated seeds found in the accept side; however, additional loss would be encountered with this additional operation,

The nine seedlots tested provided a range of seed qualities. An increase in the percent germination was recorded in eight of the nine seedlots following color sorting (Table 3). The cauliflower 'Dok Elgon' did not exhibit an improvement, even though a high percentage of seeds were discarded (Tables 2 and 3). This behavior may be attributed to the spotty fluorescence observed on the coatings of this seedlot. The greatest improvement in germination was obtained with seedlots of medium rather than of high quality. The germination of broccoli 'Shogun', 'Premium Crop', and cabbage 'Ruby Ball' increased by 10.4, 14.1, and 14.7 percentage points after sorting, respectively. The average increase in germination without or with $\mathrm{NaOCl}$ pretreatment was 5.5 and 8.2 percentage points, respectively. The $\mathrm{NaOCl}$ may have independently increased germination (Table 3), enhanced leakage (Taylor et al., 1993). and decreased fluorescence (Table 1). Further study is necessary on this pretreatment.

The system developed to upgrade seed quality in Brassica consisted of hydration, coating, drying, detection, and separation (Taylor et al., 1991). Since this system consisted of several phases, the final result of germination enhancement depends on the efficacy of each component. Nonviable seeds must first leak sinapine, and leakage is influenced by hydration conditions and seedlot. The coating must effectively trap sinapine on a single seed basis, and the coating can not be detrimental or toxic to the seeds. The color sorter must be set to discriminate between fluorescent and nonfluorescent coated seeds, and this can be performed empirically. The UV color sorter may make errors in separation: fluorescent coated seeds are not rejected or nonfluorescent-coated seeds are rejected. The upgrading of seedlots by UV color sorter was also dependent on the degree of fluorescence of the coated seeds. Coated seeds with weak or partial fluorescence may not be rejected.

In conclusion, UV color sorting technology can be effectively employed to upgrade Brassica seed quality by exploiting sinapine leakage and seed coating technology. Removal of fluorescent coated seeds by color sorting improved the subsequent percentage germination, and an increase in nonfluorescent coated seeds was correlated with an improvement in germination, $r=0.71^{* *}$. Benefits obtained from physiological seed treatments on seedling emergence can thus be improved by removal of nonviable seeds on a noninvasive basis.

\section{Literature Cited}

Association of Official Seed Analysts. 1988. Rules for testing seeds. Assn. Offic. Seed Anal. J. Seed Technol. 12(3):1-122.

Berlage, A.G., D.B. Churchil1, T.M. Cooper, and D.M. Bisland. 1989. The application of new technologies to seed conditioning. J. Agr. Eng. Res. 42:193-202.

Bouchereau, A., J. Hamelin, I. Lamour, M. Renard, and F. Larher. 1991. Distribution of sinapine and related compounds in seeds of Brassica and allied genera. Phytochemistry 30(6):1873-1881.

Bradford, K.J. 1986. Manipulation of seed water relations via osmotic priming to improve germination under stress conditions. HortScience 21:1105-1112.

Brandenburg, N.R. 1977. The principles and practice of seed cleaning: Separation with equipment that senses dimensions, shape, density, and terminal velocity of seeds. Seed Sci. \& Technol. 5:173-186.

Brandenburg, N.K. and J.K. Park. 1977. The principles and practice of seed cleaning: Separation with equipment that senses surface texture, colour, resilience, and electrical properties of seeds. Seed Sci. \& Technol. 5:187-197.

Cabrera, E.R. and J. Diaz. 1991. Removal of ballonvine (Cardiospermum halicacabum L.) seed from soybeans using an electronic colour sorting machine. Seed Sci. \& Technol. 19:203-206.

Clausen, S., L.M. Larsen, A. Ploger, and H. Sorensen. 1985. Aromatic choline esters in rapeseed, p. 61-72. In: H. Sorensen (ed.). Advances in the production and utilization of cruciferous crops. Martinus Nijhoff/Dr. W. Junk. Dordrecht.

Copeland, L.O. and M.B. McDonald. 1985. Principlesof seed science and technology. Burgess Publ. Co., Minneapolis.

Harmond, J.E., N.R. Brandenburg, and L.M. Klein. 1968. Mechanical seed cleaning and handling. U.S. Dept. of Agr., Agr Res. Serv. Agr. Hdbk. no. 354.

Hill, H.J., A.G. Taylor, and X.L. Huang. 1988. Seed viability determinations in cabbage utilizing sinapine leakage and electrical conductivity measurements. J. Expt. Bot. 39:1439-1447.

Kaufman, G. 1991. Seed coating: A tool for stand establishment: A stimulus to seed quality. HortTechnology 1:98-102.

Scott, J.M. 1989. Seed coatings and treatments and their effects on plant establishment. Adv. Agron. 42:43-83.

Taylor, A.G. and G.E. Harman. 1990. Concepts and technologies of selected seed treatments. Annu. Rev. Phytopathology 28:321-339.

Taylor, A.G., H.J. Hill, X.L. Huang, and T.G. Min. 1990. Determining seed viability. U.S. patent. no. 4,975,364.

Taylor, A.G., X.L. Huang, and H.J. Hill. 1988. Sinapine leakage from nonviable cabbage seeds. J. Expt. Bot. 39:1433-1438.

Taylor, A.G., T.G. Min, and C.A. Mallaber. 1091. Seed coating system to upgrade Brassicaceae seed quality by exploiting sinapine leakage. Seed Sci. \& Technol. 19:423-433.

Taylor, A.G., D.H. Paine, and C.A. Paine. 1993. Sinapine leakage from Brassica seeds. J. Amer. Soc. Hort. Sci. 118:546-550. 